\title{
DISTAL COMPACTIFICATIONS OF SEMIGROUPS
}

\author{
BY
}

H. D. JUNGHENN

\begin{abstract}
Properties of distal functions on semitopological semigroups are studied via the device of right topological semigroup compactification. Algebras of distal functions are used to construct the universal right simple, left simple, and group compactifications of a semigroup, and these compactifications are in turn systematically employed to obtain information about distal functions. Applications are made to semidirect products and flows.
\end{abstract}

1. Introduction. Distal functions on topological groups were studied by L. Auslander and F. Hahn in [1] and by A. Knapp in [12]. In these investigations the central theme was the connection between these functions and certain flows, the Ellis semigroup being an important device in crystalizing this connection. The purpose of the present paper is to introduce the notions of distal function, strongly distal function, and minimal distal function on an arbitrary semitopological semigroup and to study these functions from the point of view of universal semigroup compactifications. An important advantage of the compactification approach is that it provides a convenient and efficient way to study the relationship between the algebra of distal functions and related algebras of functions. For example, using compactifications one shows relatively easily that if the semigroup $S$ has a left identity or is topologically right simple, then the space of weakly almost periodic distal functions on $S$ is a tensor product of the algebra of strongly almost periodic functions on $S$ and an algebra of almost periodic functions relative to which $S$ acts as a right zero semigroup (Proposition 3.10). Another advantage of using semigroup compactifications to study distal functions is that it enables one to capitalize on the universal mapping properties that these compactifications enjoy. For example, using universal mapping properties we show in Corollary 3.9 that the space of weakly almost periodic distal functions on a semigroup $S$ is the direct sum of the algebra of strongly almost periodic functions and two ideals of weakly almost periodic "flight" functions on $S$.

The central result of this paper is Theorem 3.4, which characterizes the distal, strongly distal, and minimal distal compactifications of a semitopological semigroup $S$ in terms of universal mapping properties. A special case of Theorem 3.4 asserts that if $S$ has a left (respectively, right) identity then the distal compactification $X$ of $S$ is maximal with respect to the property that $X$ is right (respectively, left) simple.

Received by the editors October 2, 1981 and, in revised form, November 23, 1981.

1980 Mathematics Subject Classification. Primary 22A20, 43A60; Secondary 54H15.

Key words and phrases. Semitopological semigroup, distal function, strongly distal function, minimal distal function, right topological compactification, universal mapping property. 
Since the Ellis semigroup of a distal flow is always a group (even when the phase semigroup does not contain left or right identities), this result shows that use of the Ellis semigroup alone is not sufficient to reveal all of the structure of the space of distal functions on a semigroup.

In $\$ 4$ we use Theorem 3.4 to obtain general conditions under which distal functions on a dense subsemigroup extend to distal functions on the ambient semigroup (Theorems 4.3 and 4.5). The results of this section are distal analogs of known extension theorems for functions of almost periodic type. In $\$ 5$ we show that distal compactifications of certain semidirect products are again semidirect products. An obvious but important corollary of this result is that the algebra of distal functions on such a semidirect product may be expressed as a tensor product of algebras of distal functions on the factor semigroups.

The author wishes to thank Professor P. Milnes for sending him a preprint of his paper Minimal and distal functions on semidirect products of groups, which, together with the results of [12], provided the inspiration for Theorem 3.4. We also express our appreciation to the referee for suggesting several improvements to the paper.

2. Preliminaries. All topologies are assumed to be Hausdorff. If $S$ is a topological space, $C(S)$ will denote the $C^{*}$-algebra of all bounded complex-valued continuous functions on $S$. For subsets $A \subset S$ and $F \subset C(S), \mathrm{Cl}(A)$ denotes the closure of $A$ in $S$ and $\left.F\right|_{A}$ the set $\left\{\left.f\right|_{A}: f \in F\right\}$. If $T$ is a topological space and $\alpha: S \rightarrow T$ a continuous mapping then $\alpha^{*}: C(T) \rightarrow C(S)$ denotes the dual map $f \rightarrow f \circ \alpha$. The set of idempotents in a subset $B$ of a semigroup is denoted by $E(B)$.

A right topological semigroup is a semigroup $S$ with a topology relative to which the mapping $s \rightarrow s t: S \rightarrow S$ is continuous for each $t \in S$. If multiplication is separately (respectively, jointly) continuous, then $S$ is a semitopological (respectively, topological) semigroup. A right topological (respectively, semitopological) semigroup whose algebraic structure is a group is called a right topological (respectively, semitopological) group.

A compact right topological semigroup $X$ always has a smallest ideal $K(X)$, which is both the union of all of the minimal left ideals of $X$ and the union of all the minimal right ideals. The set $E=E(K(X))$ is nonempty, and the minimal left (respectively, right) ideals of $X$ are precisely the sets $X e$ (respectively, $e X$ ), where $e \in E$. If $I$ is a minimal left ideal and $J$ a minimal right ideal then $I \cap J=e X e$ for some $e \in E$. For each $e \in E, e X e$ is a (maximal) group with identity $e, E(X e)$ and $E(e X)$ are left and right zero semigroups respectively, and $K(X)$ is the union of the groups $e X e(e \in E)$. (For proofs of these and related facts see, for example, [3 or 17].)

For the remainder of this section $S$ denotes a semitopological semigroup. A subspace $F$ of $C(S)$ is left (respectively, right) translation invariant if $L(S) F \subset F$ (respectively, $R(S) F \subset F$ ), where $L(s)$ and $R(s)$ are the operators on $C(S)$ defined by $L(s) f(t)=f(s t), R(s) f(t)=f(t s)(s, t \in S ; f \in C(S))$. A sub- $C^{*}$-algebra $F$ of $C(S)$ will be called admissible if it is left and right translation invariant, contains the constant functions, and is left $m$ introverted, i.e. the function $T_{x} f(\cdot)=x(L(\cdot) f)$ is a member of $F$ whenever $f \in F$ and $x \in \operatorname{spec}(F)=$ space of nonzero continuous 
complex homomorphisms on $F$. In this case $T_{x}: F \rightarrow F$ is called the left $m$ introversion operator determined by $x$. Let $F$ be admissible, $X=\operatorname{spec}(F)$ with the relativized weak* topology, and $\alpha: S \rightarrow X$ the evaluation mapping $(\alpha(s) f=f(s) ; s \in S$, $f \in F)$. Define multiplication in $X$ by $x y=x \circ T_{y}$. Then the pair $(X, \alpha)$, called the canonical $F$ compactification of $S$, has the following properties (for details see [3, I.4]):

(a) $X$ is a compact right topological semigroup;

(b) $\alpha: S \rightarrow X$ is a continuous homomorphism with dense range such that the mapping $x \rightarrow \alpha(s) x: X \rightarrow X$ is continuous for each $s \in S$;

(c) $\alpha^{*}(C(X))=F$;

(d) $[\alpha(s) x](f)=x(L(s) f),[x \alpha(s)](f)=x(R(s) f)(f \in F, s \in S, x \in X)$;

(e) $x \rightarrow T_{x}$ is a representation of the semigroup $X$ by bounded linear operators on $F$;

(f) for each $f \in F,\left\{T_{x} f: x \in X\right\}=\mathrm{Cl}(R(S) f)$ (closure in the topology of pointwise convergence on $C(S))$.

Any pair $(X, \alpha)$ satisfying (a) and (b) will be called a right topological compactification of $S$, and an $F$ compactification if in addition (c) holds. For a fixed admissible subalgebra $F$ of $C(S)$, all $F$ compactifications of $S$ are (algebraically and topologically) isomorphic (and hence we are justified in speaking of the $F$ compactification of $S$ ). This fact may be deduced from the following lemma, which is implicit in the development of §III.2 of [3] and which will be useful in $\$ 3$ below. We omit the straightforward proof.

2.1. LEMMA. Let $S$ be a semitopological semigroup, $F$ an admissible subalgebra of $C(S),(X, \alpha)$ an $F$ compactification of $S$, and $(Y, \beta)$ a right topological compactification of $S$. Then $\beta^{*}(C(Y)) \subset F$ if and only if there exists a continuous homomorphism $\gamma$ : $X \rightarrow Y$ such that $\gamma \circ \alpha=\beta$.

A right topological compactification $(X, \alpha)$ of $S$ is said to be maximal with respect to a property $\mathrm{P}$ if $(X, \alpha)$ has property $\mathrm{P}$, and whenever $(Y, \beta)$ is a right topological compactification of $S$ with property $\mathrm{P}$ then there exists a continuous homomorphism $\gamma: X \rightarrow Y$ such that $\gamma \circ \alpha=\beta$. Thus $F$ compactifications are maximal with respect to the property that $\alpha^{*}(C(X)) \subset F$. The factorization of the mapping $\beta$ by $\alpha$ is sometimes referred to as a universal mapping property of $(X, \alpha)$.

The following examples of admissible subalgebras of $C(S)$ will be needed in the sequel:

$$
\begin{aligned}
\operatorname{LMC}(S)= & \{f \in C(S): L(\cdot) f \text { is } \sigma(C(S)), \operatorname{spec}(C(S)) \text { continuous }\}, \\
L U C(S)= & \{f \in C(S): L(\cdot) f \text { is norm continuous }\}, \\
W A P(S)= & \{f \in C(S): R(S) f \text { is relatively weakly compact }\}, \\
A P(S)= & \{f \in C(S): R(S) f \text { is relatively norm compact }\}, \\
\operatorname{SAP}(S)= & \text { closed linear span in } C(S) \text { of the coefficients } \\
& \text { of all continuous finite dimensional unitary representations of } S .
\end{aligned}
$$


We shall occasionally suppress the letter $S$ from the notation for these algebras. For a detailed description of the above algebras and for the universal mapping properties of the associated compactifications the reader is referred to Chapter III of [3].

A flow is a triple $(S, Z, \pi)$, where $S$ is a semitopological semigroup, $Z$ is a compact topological space and $\pi: S \rightarrow Z^{Z}$ is a continuous homomorphism such that $\pi(s): Z \rightarrow Z$ is continuous for each $s \in S$. Here $Z^{Z}$ denotes the compact right topological semigroup (with respect to the product topology and function composition) of all self-maps of $Z$. The symbol $\pi$ is frequently suppressed from the notation $(S, Z, \pi)$. As is customary we shall write $s z$ for $\pi(s)(z)$ and $S z$ for the set $\{s z$ : $s \in S\}$.

A flow $(S, Z)$ is jointly continuous if the mapping $(s, z) \rightarrow s z: S \times Z \rightarrow Z$ is continuous, point transitive if $S z$ is dense in $Z$ for some $z \in Z$, and distal if whenever $x$ and $y$ are members of $Z$ such that $\lim _{k} s_{k} x=\lim _{k} s_{k} y$ for some net $\left(s_{k}\right)$ in $S$ then $x=y$. Thus $(S, Z)$ is distal if and only if each member of $E(S, Z)$ is injective, where $E(S, Z)$ (the Ellis semigroup of $(S, Z)$ ) denotes the closure of $\pi(S)$ in $Z^{Z}$. Using the structure theory of compact right topological semigroups outlined in the beginning of this section- one may readily prove the following result of $\mathrm{R}$. Ellis: $(S, Z)$ is distal if and only if $E(S, Z)$ is a group (with respect to function composition and with identity the identity function) [5, Proposition 5.3].

3. Distal functions on semigroups. Let $S$ be a semitopological semigroup, $f \in$ $L M C(S)$, and $Z=Z_{f}$ the closure of $R(S) f$ in the topology of pointwise convergence on $C(S)$. Define $\pi: S \rightarrow Z^{Z}$ by $\pi(s)=\left.R(s)\right|_{Z}$. Then $Z$ is (pointwise) compact [14], and $(S, Z, \pi)$ is easily seen to be a flow. If this flow is distal then $f$ is said to be a distal function. The set of all distal functions on $S$ is denoted by $D(S)$. We shall write $D L U C(S)$ for $D(S) \cap L U C(S)$. If $S$ is a topological group then $D L U C(S)$ is the class of distal functions as defined in [12 and 15].

A member $f$ of $D(S)$ is said to be minimal if $g \in Z_{f}$ implies $f \in Z_{g}$. We shall denote the set of all minimal functions in $D(S)$ by $M D(S)$ and write $M D L U C(S)$ for $M D(S) \cap L U C(S)$.

Define the semitopological semigroup $S^{1}$ as follows: $S^{1}=S$ if $S$ has an identity, otherwise $S^{1}$ is the semigroup $S \cup\{1\}$ obtained by adjoining a discrete identity to $S$. If $f \in C(S)$ define $f^{1} \in C\left(S^{1}\right)$ so that $\left.f^{1}\right|_{S}=f$ and (if $S$ has no identity) $f^{1}(1)=0$. Let $f \in L M C(S)$ and let $Z=Z_{f^{1}}$ denote the pointwise closure of $R(S) f^{1}$ in $C\left(S^{1}\right)$. Define $\pi^{1}: S \rightarrow Z^{Z}$ by $\pi^{1}(s)=\left.R(s)\right|_{Z}(s \in S)$. Then $Z$ is compact in the topology of pointwise convergence on $C\left(S^{1}\right)$, and $\left(S, Z, \pi^{1}\right)$ is a flow. We shall say that $f$ is strongly distal if the flow $\left(S, Z, \pi^{1}\right)$ is distal. The set of all strongly distal functions on $S$ will be denoted by $S D(S)$. Also, we define $S D L U C(S)=S D(S) \cap L U C(S)$.

3.1. Remarks. Let $(X, \alpha)$ denote the canonical $L M C$ compactification of $S$ and let $f \in L M C(S)$. From property (f) of $\S 2$ we have $Z_{f}=\left\{T_{x} f: x \in X\right\}$, where $T_{x}$ is the left $m$ introversion operator on $L M C(S)$ determined by $x$. It is easily verified that the mapping $\zeta: X \rightarrow E\left(S, Z_{f}\right)$ defined by $\zeta(x)\left(T_{y} f\right)=T_{x y} f$ is a well-defined continuous homomorphism of $X$ onto $E\left(S, Z_{f}\right)$ satisfying $\zeta \circ \alpha=\pi$. In particular we see that $f \in D(S)$ if and only if $\zeta(x)$ is injective for each $x \in X$, i.e. if and only if 
$T_{x y} f=T_{x z} f$ implies $T_{y} f=T_{z} f$. Similarly, if we define $T_{x}^{1} f$ on $S^{1}$ by $T_{x}^{1} f(s)=$ $x(L(s) f)(s \in S)$ and (if $S$ has no identity) $\left(T_{x}^{1} f\right)(1)=x(f)$, then $Z_{f^{\prime}}=\left\{T_{x}^{1} f\right.$ : $x \in X\}$ and the mapping $\zeta^{1}: X \rightarrow E\left(S, Z_{f^{\prime}}\right)$ defined by $\zeta^{1}(x)\left(T_{y}^{1} f\right)=T_{x y}^{1} f$ is a well-defined continuous homomorphism onto $E\left(S, Z_{f^{\prime}}\right)$ such that $\zeta^{1} \circ \alpha=\pi^{1}$. Also, from the definition of $M D(S)$ we see that $f \in M D(S)$ if and only if for each $y \in X$ there exists some $x \in X$ (depending on $y$ and $f$ ) such that $T_{x y} f=f$.

Part (i) of the following lemma was proved by A. Knapp for the case $S$ a topological group [12, Theorem 3.7]. We give a simple proof based on Remark 3.1.

3.2. Lemma. Let $S$ be a semitopological semigroup, $(X, \alpha)$ the canonical LMC compactification of $S$, and $f \in L M C(S)$.

(i) The following statements are equivalent:

(a) $f \in D(S)$.

(b) $($ uev $)(f)=(u v)(f)$ for all $u, v \in X$ and $e \in E(X)$.

(c) There exists some $e \in E(K(X))$ such that $(u e v)(f)=(u v)(f)$ for all $u$, $v \in X$.

(ii) The following statements are equivalent:

(a) $f \in S D(S)$.

(b) $f \in D(S)$ and $(e v)(f)=v(f)$ for all $v \in X$ and $e \in E(X)$. $v \in X$.

(c) $f \in D(S)$ and there exists $e \in E(K(X))$ such that $(e v)(f)=v(f)$ for all

(iii) The following statements are equivalent:

(a) $f \in M D(S)$.

(b) $f \in D(S)$ and $(v e)(f)=v(f)$ for all $v \in X$ and $e \in E(X)$.

(c) $f \in D(S)$ and there exists $e \in E(K(X))$ such that $(v e)(f)=v(f)$ for all $v \in X$.

Proof. (i) If $f \in D(S)$, then for all $v \in X$ and $e \in E(X) T_{e v} f=T_{e e v} f$; hence, by 3.1, $T_{v} f=T_{e v} f$. Thus (a) $\Rightarrow(\mathrm{b})$. That (b) $\Rightarrow$ (c) is obvious. Assume that (c) holds and let $\zeta: X \rightarrow E\left(S, Z_{f}\right)$ be the map defined in 3.1. For all $v \in X$ we have $\zeta(e)\left(T_{v} f\right)=$ $T_{e v} f=T_{v} f$; hence $\zeta(e)$ is the identity mapping. Since $e X e$ is a group so is $\zeta(e X e)=\zeta(X)=E\left(S, Z_{f}\right)$. Therefore $f \in D(S)$. The proof of (ii) is similar.

(iii) Let $f \in M D(S)$ and $e \in E(X)$. Choose $x \in X$ such that $T_{x e} f=f$. Then for all $v \in X,(v x e)(f)=v(f)$, so by (i)(b) $v(f)=($ vexe $)(f)=(v e)(f)$. Thus (a) $\Rightarrow$ (b). Clearly (b) $\Rightarrow$ (c). Assume that (c) holds. Since $e \in E(K(X))$, given $y \in X$ there exists $x \in X$ such that $x e y e=e$. Then by (i)(b) and hypothesis, $T_{x y} f=T_{x y} T_{e} f=$ $T_{\text {xye }} f=T_{\text {xeye }} f=T_{e} f=f$; hence $f \in M D(S)$. This completes the proof.

A homomorphism $r$ of a semigroup $Y$ onto a subsemigroup $Z$ is called a retraction if $r: Z \rightarrow Z$ is the identity function. If also $Y^{2} \subset Z$ then $r$ is called an inflation function and $Y$ an inflation of the semigroup $Z$ (see, for example, [18]). A semigroup $U$ is called a rectangular group if $U$ is algebraically isomorphic to a direct product semigroup $L \times G \times R$, where $L$ is a left zero semigroup, $R$ a right zero semigroup and $G$ is a group. If $L$ (respectively, $R$ ) is the trivial semigroup then $U$ is called a right group (respectively, left group). We remark that in the presence of topologies on $Y$ or $U$ we do not require any of the mappings in these definitions to be continuous. 
3.3. LemmA. Let $Y$ be a compact right topological semigroup and $K=K(Y)$. The following statements are equivalent:

(a) $Y$ is an inflation of a rectangular group.

(b) $x e y=x y$ for all $x, y \in Y$ and all $e \in E(Y)$.

(c) $Y^{2}=K$, and for each $e \in E(Y)$ (equivalently, for some $e \in E(Y)$ ) the mappings $f_{e}: y \rightarrow y(\text { eye })^{-1}, g_{e}: y \rightarrow$ eye and $h_{e}: y \rightarrow(e y e)^{-1} y$ are restrictions of $Y$ onto $E(Y e)$, $e Y e$ and $E(e Y)$ respectively, where (eye $)^{-1}$ denotes the inverse of eye in the group eYe.

If these conditions hold then $K$ is a rectangular group and $Y$ is an inflation of $K$ (and only of $K$ ).

Proof. (a) $\Rightarrow(\mathrm{b})$. Let $Y$ be an inflation of the rectangular group $Z$ and $r: Y \rightarrow Z$ the inflation function. Since (b) holds in the semigroup $Z$ we have $x e y=$ $r(x) r(e) r(y)=r(x) r(y)=x y$ for all $x, y \in Y$ and $e \in E(Y)$. Note that $K \subset Y^{2} \subset$ $Z$ and $Z$ simple imply that $K=Z$. Hence, $Y$ can only be an inflation of $K$.

(b) $\Rightarrow$ (c). This implication is clear.

(c) $\Rightarrow$ (a). Assume (c) holds for some $e \in E(Y)$, and define $r: Y \rightarrow K$ by $r(y)=$ $f_{e}(y) g_{e}(y) h_{e}(y)=y(e y e)^{-1} y$. It is well known that in general the mapping ' $k$ : $y \rightarrow\left(f_{e}(y), g_{e}(y), h_{e}(y)\right)$ is a bjection from $K$ onto the direct product semigroup $E(Y e) \times e Y e \times E(e Y)$, with $k^{-1}(u, v, w)=u v w$ (see, for example, [3, p. 51]). Since $f_{e}, g_{e}$ and $h_{e}$ are homomorphisms, so is $k$. Thus $K$ is a rectangular group, and for $y \in K$ we have $y=k^{-1}(k(y))=r(y)$. Finally, if $x, y \in Y$ then

$$
\begin{aligned}
r(x y) & =f_{e}(x y) g_{e}(x y) h_{e}(x y)=f_{e}(x) g_{e}(x) g_{e}(y) h_{e}(y) \\
= & f_{e}(x) g_{e}(x) e g_{e}(y) h_{e}(y)=f_{e}(x) g_{e}(x) h_{e}(x) f_{e}(y) g_{e}(y) h_{e}(y)=r(x) r(y),
\end{aligned}
$$

where we have used the obvious identities $f_{e}(x y)=f_{e}(x), h_{e}(x y)=h_{e}(y)$ and $h_{e}(x) f_{e}(y)=e$.

P. Milnes has observed that if $S$ is a topological group then $D(S)$ is admissible and the $D$ compactification $(Y, \beta)$ of $S$ is maximal with respect to the property that $Y$ is a group [15]. More generally we have the following result:

3.4. TheOrem. Let $S$ be a semitopological semigroup. Then $D(S), S D(S), M D(S)$ and $S D(S) \cap M D(S)$ are admissible subalgebras of $C(S)$, and the $D, S D, M D$, and $S D \cap M D$ compactifications $(Y, \beta)$ of $S$ are maximal, respectively, with respect to the following properties:

(a) $Y$ is an inflation of a rectangular group.

(b) $Y$ is right simple (equivalently, $Y$ is a right group).

(c) $Y$ is left simple (equivalently, $Y$ is a left group).

(d) $Y$ is a group.

Proof. That $D(S)$ is admissible is immediate from 3.2. Let $(X, \alpha)$ and $(Y, \beta)$ denote respectively the canonical $L M C$ and $D$ compactifications of $S$ and let $\theta$ : $X \rightarrow Y$ denote the restriction mapping $\left(\theta(x)=\left.x\right|_{D(S)}\right)$. Then $\theta$ is a continuous homomorphism onto $Y$ satisfying $\theta \circ \alpha=\beta$, as is easily verified. Given $x, y \in Y$ and $e \in E(Y)$ choose $u, v \in X$ and $d \in E(X)$ such that $\theta(u)=x, \theta(v)=y$ and $\theta(d)=e$ (the choice of $d$ is possible because $\theta^{-1}(e)$ is a compact subsemigroup of 
$X)$. Then for any $f \in D(S),(x e y)(f)=\theta(u d v)(f)=(u d v)(f)=(u v)(f)=$ $(x y)(f)$, the third equality by 3.2. Thus by Lemma $3.3 Y$ has property (a). To prove that $(Y, \beta)$ is maximal with respect to this property it remains to show that $\beta_{0}^{*}\left(C\left(Y_{0}\right)\right) \subset D(S)$ for any right topological compactification $\left(Y_{0}, \beta_{0}\right)$ having property 3.3(b) (Lemma 2.1). Let $g \in C\left(Y_{0}\right)$ and set $f=\beta_{0}^{*}(g)$. If $w \in \operatorname{spec}(C(S))$, then $w \circ \beta_{0}^{*}$ is in the spectrum of $C\left(Y_{0}\right)$, and therefore $w \circ \beta_{0}^{*}$ is evaluation at some point $y \in Y_{0}$. From the identity $L(s) f=\beta_{0}^{*}\left(L\left(\beta_{0}(s)\right) g\right)$ we have $w(L(s) f)=g\left(\beta_{0}(s) f\right)$ $(s \in S)$; hence $f \in L M C(S)$. Now, by 2.1 , there exists a continuous homomorphism $\delta: X \rightarrow Y_{0}$ such that $\beta_{0}=\delta \circ \alpha$. Then if $u, v \in X$ and $e \in E(X)$ we have $(u e v)(f)$ $=g(\delta(u e v))=g(\delta(u v))=(u v)(f)$; hence, by $3.2, f \in D(S)$.

That $S D(S)$ is admissible follows from 3.2. Also, the proof of the first part of the theorem is easily modified to show that the $S D$ compactification $(Y, \beta)$ of $S$ is maximal with respect to the property that $e y=y$ for all $y \in Y$ and $e \in E(Y)$. But this property is equivalent to right simplicity of $Y$, which is equivalent to the property that $Y$ is a right group (see, for example, [3, p. 35]). The proofs for $M D(S)$ and $S D(S) \cap M D(S)$ are similar.

3.5. Corollary. (a) If $\mathrm{Cl}(s S)=S$ for some $s \in S$, then $D(S)=S D(S)$.

(b) If $\mathrm{Cl}(S t)=S$ for some $t \in S$, then $D(S)=M D(S)$.

Proof. (a) If $\mathrm{Cl}(s S)=S$ for some $s \in S$ and if $(Y, \beta)$ denotes the $D$ compactification of $S$, then $K(Y) \supset Y^{2} \supset \beta(s) Y=Y$ and hence there exists $e \in E(Y)$ such that $e \beta(s)=\beta(s)$ (see §2). Then $Y=\beta(s) Y=e \beta(s) Y \subset e Y$, which shows that $Y$ is right simple. It follows easily from 3.4 that $D(S)=S D(S)$. The proof of (b) is similar.

3.6. Corollary. (a) $S D(S) \cap W A P(S)=S D(S) \cap A P(S)$.

(b) $M D(S) \cap W A P(S)=M D(S) \cap A P(S)$.

(c) $S D(S) \cap M D(S) \cap W A P(S)=S A P(S)$.

Proof. Let $(Z, \gamma)$ denote the $S D \cap W A P$ compactification of $S$. Then $Z$ is a continuous homomorphic image of the $S D$ compactification of $S$ and is therefore right simple by 3.4. Also, $Z$ is semitopological; hence by Ellis's theorem [6], $Z$ is a topological semigroup [2, p. 60]. It follows that $S D(S) \cap W A P(S) \subset A P(S)$ (see [3, III.9.5]). This proves (a). The proofs of (b) and (c) are similar.

For an admissible subalgebra $F$ of $C(S)$ we shall denote by $F_{0 r}$ (respectively, $F_{0 l}$ ) the set of all $f$ in $F$ with the property that $\mathrm{Cl}(R(S) f)$ (respectively, $\operatorname{Cl}(L(S) f)$ ) contains the zero function, where the closures are in the pointwise topology of $C(S)$. It is easily verified that $F_{0 r}$ (respectively, $F_{0 l}$ ) has all of the properties of a left (respectively, right) translation invariant norm closed conjugate closed proper ideal of $F$ except possibly closure under addition.

The following corollary is a generalization of the well-known result of $\mathrm{K}$. de Leeuw and I. Glicksberg which asserts that (for a semigroup $S$ with identity) $W A P(S)=S A P(S) \oplus W A P(S)_{0 r}$ if and only if the WAP compactification of $S$ has unique minimal left and right ideals [4, Theorem 5.7]. 
3.7. COROLlaRY. Let $S$ be a semitopological semigroup, $F$ an admissible subalgebra of $C(S)$, and $(X, \alpha)$ the $F$ compactification of $S$.

(a) $F=[M D(S) \cap F] \oplus H$ for some left translation invariant norm closed conjugate closed ideal $H$ of $F$ contained in $F_{0 r}$ if and only if there exists $e \in E(K(X))$ such that

$$
\text { xeye }=\text { xye } \quad(x, y \in X) \text {. }
$$

(b) $F_{0 r}$ is an ideal of $F$ and $F=[M D(S) \cap F] \oplus F_{0 r}$ if and only if $X$ has a unique minimal left ideal and there exists $e \in E(K(X))$ such that (1) holds.

(c) $F=[S D(S) \cap M D(S) \cap F] \oplus H$ for some left translation invariant norm closed conjugate closed ideal $H$ of $F$ contained in $F_{0 r}$ if and only if $X$ has a unique minimal right ideal. In this case there exists a left invariant mean $\mu$ on $F$ such that $H=\{f \in F: \mu(|f|)=0\}$.

(d) $F_{0 r}$ is an ideal of $F$ and $F=[S D(S) \cap M D(S) \cap F] \oplus F_{0 r}$ if and only if $X$ has unique minimal left and right ideals.

(e) If $F \subset W A P(S)$, then $F=[S D(S) \cap F] \oplus H$ for some right translation invariant norm closed conjugate closed ideal $H$ of $F$ contained in $F_{0 l}$ if and only if there exists $e \in E(K(X))$ such that

$$
\text { exey }=\text { exy } \quad(x, y \in X) .
$$

(f) If $F \subset W A P(S)$ then $F_{0 l}$ is an ideal of $F$ and $F=[S D(S) \cap F] \oplus F_{0}$ if and only if $X$ has a unique minimal left ideal and there exists $e \in E(K(X))$ such that (2) holds.

Proof [sufficiency of (a)]. Let $B=M D(S) \cap F$ and let $(V, \delta)$ denote the canonical $B$ compactification of $S$. We may assume that $(X, \alpha)$ is canonical. Condition (1) implies that $\rho: x \rightarrow x e: X \rightarrow X e$ is a continuous homomorphism. Since $X e$ is left simple it follows from 2.1 and 3.4 that $(\rho \circ \alpha)^{*}(C(X e)) \subset M D(S)$. Also, $(\rho \circ \alpha)^{*}(C(X e)) \subset \alpha^{*}(C(X))=F$; hence $(\rho \circ \alpha)^{*}(C(X e)) \subset B$. We wish to show that this last inclusion is actually equality. By 2.1 there exists a continuous surjective homomorphism $\psi: V \rightarrow X e$ such that $\rho \circ \alpha=\psi \circ \delta$. The restriction mapping $\theta$ : $X \rightarrow V$ is a continuous homomorphism onto $V$ such that $\theta \circ \alpha=\delta$, hence $\rho \circ \alpha=$ $\psi \circ \theta \circ \alpha$ and therefore $\rho=\psi \circ \theta$. Since $V$ is a homomorphic image of the $M D$ compactification of $S, V$ must be left simple and so $\theta \circ \psi \circ \theta=\theta \circ \rho=\theta$. Therefore $\theta \circ \psi$ is the identity mapping on $V$ and hence $\psi$ must be a bijection and $(\rho \circ \alpha)^{*}=$ $\delta^{*} \circ \psi^{*}$ an isometric isomorphism from $C(X e)$ onto $B$. Let $T_{e}$ denote the left $m$ introversion operator on $F$ determined by $e$. If $f \in F$ and $\alpha^{*}(\hat{f})=f$, then $T_{e} f=$ $(\rho \circ \alpha)^{*}\left(\left.\hat{f}\right|_{X e}\right)$; hence by Tietze's extension theorem $T_{e}(F)=(\rho \circ \alpha)^{*}(C(X e))=B$. Since $T_{e}$ is a projection, $F=B \oplus H$, where $H=\operatorname{ker}\left(T_{e}\right)$. That $H$ has the required properties is readily verified.

[Sufficiency of (b)]. By the preceding it suffices to show that if $X$ also has a unique minimal left ideal, then $F_{0 r} \subset H$. Let $f \in F_{0 r}$. Then by property (f) in $\$ 2$ there exists $x \in X$ such that $T_{x} f=0$. The set of all such $x$ is a closed left ideal of $X$ and hence must contain $e$. Therefore $f \in \operatorname{ker}\left(T_{e}\right)=H$. 
[Necessity of (a)]. Let $P$ denote the projection of $F$ onto $B=M D(S) \cap F$. Then $H=\operatorname{ker}(P)$ and

$$
P^{*}(V)=\{x \in X: x(H)=\{0\}\},
$$

where $P^{*}: B^{*} \rightarrow F^{*}$ denotes the dual operator and $(V, \delta)$ is the canonical $B$ compactification of $S$. Now, it follows easily from the fact that $P(F)$ is left translation invariant and left $m$ introverted that $\left.P^{*}\right|_{V}$ is a homomorphism and hence $P^{*}(V)$ is a closed left simple subsemigroup of $X$. Since $H$ is left translation invariant, (3) shows that $P^{*}(V)$ is also a left ideal of $X$. Therefore $P^{*}(V)$ must be a minimal left ideal and so

$$
P^{*}(V)=X e
$$

for some $e \in E(K(X))$. Let $\theta: X \rightarrow V$ denote the restriction mapping. For any $x \in X$ and $f \in F, x\left(P T_{e} f\right)=\theta(x)\left(P T_{e} f\right)=P^{*}(\theta(x))\left(T_{e} f\right)=\left[P^{*}(\theta(x)) e\right](f)=$ $\left[P^{*}(\theta(x))\right](f)=x(P f)$; hence $P T_{e} f=P f$. Therefore $T_{e} f-P f \in H$, so from (3) and (4) we have $(x e)\left(T_{e} f\right)=(x e)(P f)$. But $(x e)\left(T_{e} f\right)=x\left(T_{e} T_{e} f\right)=(x e)(f)$ and $(x e)(P f)=P^{*}(\theta(x e))(f)=P^{*}(\theta(x) \theta(e))(f)=P^{*}(\theta(x))(f)$, the last equality because $V$ is left simple. Therefore we have $P^{*}(\theta(x))=x e$ for all $x \in X$. Since $P^{*} \circ \theta$ is a homomorphism, (1) holds.

[Necessity of (b)]. We must show that if in the preceding argument $H=F_{0 r}$ then $X e$ is the unique minimal left ideal of $X$. If $I$ were a minimal left ideal distinct from $X e$ then $I \cap X e=\varnothing$ and hence (since $I$ and $X e$ are closed) there would exist $f \in F$ such that $\hat{f}(I)=\{0\}$ and $\hat{f}(X e)=\{1\}$, where $\alpha^{*}(\hat{f})=f$. But then for any $x \in I$ and $s \in S,\left(T_{x} f\right)(s)=\hat{f}(\alpha(s) x)=0$; hence $f \in F_{0 r}=\operatorname{ker}(P)$ and therefore $\hat{f}(X e)$ $=\hat{f}\left(P^{*}(V)\right)=V(P f)=\{0\}$, a contradiction. Thus $X e$ is unique.

[Sufficiency of (c)]. If $X$ has a unique minimal right ideal then, for any $e \in$ $E(K(X)), X e=e X e$, a group, and arguing as in sufficiency of (a), taking $B=$ $S D(S) \cap M D(S) \cap F$, we obtain $F=B \oplus H$ where $H=\operatorname{ker}\left(T_{e}\right)$. Let $\nu$ be a probability measure on $\mathrm{Xe}$ such that

$$
\int h(x y) \nu(d y)=\int h(y) \nu(d y)
$$

for all $x \in \alpha(S) e$ and $h \in C(X e)$ (see [7 or 16]). The equation $\mu(f)=\left.\int \hat{f}\right|_{X e} d \nu$ $\left(f \in F, \alpha^{*}(\hat{f})=f\right)$ then defines a left invariant mean $\mu$ on $F$. Since $\mu(|f|)=0 \Leftrightarrow \hat{f}$ $=0$ on $X e \Leftrightarrow T_{e} f=0$ we see that $H=\{f \in F: \mu(|f|)=0\}$.

[Necessity of (c)]. Argue as in necessity of (a) taking $B=S D(S) \cap M D(S) \cap F$ and $(V, \delta)$ the canonical $B$ compactification of $S$. Since now $V$ is a group, by (4) $X e=e X e$ for some $e \in E(K(X))$. For any $d \in E(K(X)), X e \cap d X$ is a maximal subgroup of $e X e$ and therefore must coincide with $e X e$ (see §2). Thus $e X e \subset d X \cap e X$ so by minimality, $d X=e X$. Therefore $X$ has a unique minimal right ideal. The proofs of (d), (e) and (f) are similar respectively to the proofs of (b), (a) and (b).

3.8. Corollary. $S D(S)$ has a left invariant mean, and there exist left translation invariant norm closed conjugate closed ideals $H_{1}$ of $D(S)$ and $H_{2}$ of $S D(S)$ such that $D(S)=M D(S) \oplus H_{1}$ and $S D(S)=[S D(S) \cap M D(S)] \oplus H_{2}$, where $H_{1} \subset D(S)_{0 r}$ and $H_{2} \subset S D(S)_{0 r}$. 
3.9. COROLlaRY. There exist norm closed conjugate closed ideals $H$ of $W A P(S) \cap$ $D(S)$ and $G$ of $A P(S) \cap M D(S)$ such that $H$ is left translation invariant, $G$ is right translation invariant, $H \subset[W A P(S) \cap D(S)]_{0 r}, \quad G \subset[A P(S) \cap M D(S)]_{0 l}$ and $W A P(S) \cap D(S)=S A P(S) \oplus G \oplus H$. A similar result holds with $M D(S)$ replaced by $S D(S)$ (and all right and left notions interchanged).

Proof. By 3.7(a) applied to $W A P(S) \cap D(S)$ and by 3.6(b) we have $W A P(S) \cap$ $D(S)=[A P(S) \cap M D(S)] \oplus H$, where $H$ has the required properties. To complete the proof of the $M D$ case apply 3.7(e) to $F=A P(S) \cap M D(S)$ and use 3.6(c). The proof of the $S D$ case is similar.

Let $L Z(S)$ denote the set of all functions in $C(S)$ relative to which $S$ behaves like a left zero semigroup. More precisely,

$$
L Z(S)=\{f \in C(S): f(s t)=f(s) \text { for all } s, t \in S\} .
$$

Similarly, define

$$
R Z(S)=\{f \in C(S): f(s t)=f(t) \text { for all } s, t \in S\} .
$$

Clearly, $L Z(S)$ and $R Z(S)$ are admissible subalgebras of $A P(S)$ with associated compactifications which are maximal with respect to the properties of being left zero and right zero semigroups respectively. In particular, by 3.4, $L Z(S) \subset M D(S)$ and $R Z(S) \subset S D(S)$.

If $F$ is a subset of $C(S)$ and $A$ and $B$ are subspaces then $\langle F\rangle$ will denote the $C^{*}$ subalgebra of $C(S)$ generated by $F$ and $A \otimes B$ the topological tensor product of $A$ and $B$ (i.e. the completion in the least cross norm of the algebraic tensor product).

3.10. Proposition. Let $S$ be a semitopological semigroup and let $\left(X_{i}, \alpha_{i}\right)(i=1,2,3)$ denote, respectively, the $L Z, S A P$, and $R Z$ compactifications of $S$.

(a) If $B=\langle L Z(S) \cup S A P(S) \cup R Z(S)\rangle$ then $B$ is admissible, and $\left(X_{1} \times X_{2} \times X_{3}\right.$, $\left.\left(\alpha_{1}, \alpha_{2}, \alpha_{3}\right)\right)$ is a $B$ compactification of $S$, where $X=X_{1} \times X_{2} \times X_{3}$ is the direct product semigroup and $\alpha=\left(\alpha_{1}, \alpha_{2}, \alpha_{3}\right)$ is defined by $\alpha(s)=\left(\alpha_{1}(s), \alpha_{2}(s), \alpha_{3}(s)\right)$. Furthermore, $B=L Z(S) \otimes S A P(S) \otimes R Z(S)$, and $(X, \alpha)$ is maximal with respect to the property that $X$ is a compact topological rectangular group. Also, if $\mathrm{Cl}\left(S^{2}\right)=S$ then $B=D(S) \cap A P(S)$.

(b) $S D(S) \cap A P(S)=\langle S A P(S) \cup R Z(S)\rangle=S A P(S) \otimes R Z(S)$, and $\left(X_{2} \times\right.$ $\left.X_{3},\left(\alpha_{2}, \alpha_{3}\right)\right)$ is an $S D \cap A P$ compactification of $S$.

(c) $M D(S) \cap A P(S)=\langle L Z(S) \cup S A P(S)\rangle=L Z(S) \otimes S A P(S)$, and $\left(X_{1} \times\right.$ $\left.X_{2},\left(\alpha_{1}, \alpha_{2}\right)\right)$ is an $M D \cap A P$ compactification of $S$.

Proof. We prove only (a); the proofs of (b) and (c) are similar. We show first that $\alpha(S)$ is dense in $X$. Let $(W, \delta)$ denote the $L M C$ compactification of $S$ and for each $i$ let $\theta_{i}: W \rightarrow X_{i}$ be a continuous homomorphism onto $X_{i}$ such that $\theta_{i} \circ \delta=\alpha_{i}$. The existence of $\alpha_{i}$ is a consequence of the universal mapping property of $L M C$ compactifications (see, for example, [3, III.4]). Define $\theta: W \rightarrow X$ by $\theta(w)=$ $\left(\theta_{1}(w), \theta_{2}(w), \theta_{3}(w)\right)$. For any $u, v, w \in W$ we have

$$
\theta(u v w)=\left(\theta_{1}(u), \theta_{2}(u v w), \theta_{3}(w)\right),
$$

and since $X_{2}$ is a group it follows that $\theta$ is surjective. Therefore, since $\theta \circ \delta=$ $\alpha, \mathrm{Cl}(\alpha(S))=X$. 
To see that $\alpha^{*}(C(X))=B$ simply note that $\alpha^{*}(C(X))$ is generated by all functions of the form

$$
\alpha^{*}\left(g_{1} \otimes g_{2} \otimes g_{3}\right)=\alpha_{1}^{*}\left(g_{1}\right) \alpha_{2}^{*}\left(g_{2}\right) \alpha_{3}^{*}\left(g_{3}\right) \quad\left(g_{i} \in C\left(X_{i}\right)\right) .
$$

Thus $(X, \alpha)$ is a $B$ compactification of $S$. That $(X, \alpha)$ is maximal with respect to the property that $X$ is a compact topological rectangular group is an easy consequence of the universal properties of $\left(X_{i}, \alpha_{i}\right)$. It follows that if $\operatorname{Cl}\left(S^{2}\right)=S$ (so that the $D \cap A P$ compactification of $S$ is simple and hence a rectangular group) then $B=D(S) \cap A P(S)$. Finally to see that $B$ may be written as a tensor product, let $f_{j} \in R Z(S), g_{j} \in S A P(S)$, and $h_{j} \in L Z(S)(j=1, \ldots, n)$ and choose $\hat{f}_{j}$, $\hat{g}_{j}$, and $\hat{h}_{j}$ such that $\alpha_{1}^{*}\left(f_{j}\right)=f_{j}, \alpha_{2}^{*}\left(\hat{g}_{j}\right)=g_{j}$, and $\alpha_{3}^{*}\left(\hat{h}_{j}\right)=h_{j}$. Then,

$$
\begin{aligned}
\left\|\sum_{j=1}^{n} f_{j} \otimes g_{j} \otimes h_{j}\right\| & =\sup _{x_{i} \in X_{i}}\left|\sum_{j} \hat{f}_{j}\left(x_{1}\right) \hat{g}_{j}\left(x_{2}\right) \hat{h}_{j}\left(x_{3}\right)\right| \\
& =\sup _{u, v, w \in W}\left|\sum_{j} \hat{f}_{j}\left(\theta_{1}(u)\right) \hat{g}_{j}\left(\theta_{2}(v)\right) \hat{h}_{j}\left(\theta_{3}(w)\right)\right| \\
& =\sup _{u, v, w \in W}\left|\sum_{j} \hat{f}_{j}\left(\theta_{1}(u v w)\right) \hat{g}_{j}\left(\theta_{2}(u v w)\right) \hat{h}_{j}\left(\theta_{3}(u v w)\right)\right| \\
& =\left\|\sum_{j} f_{j} g_{j} h_{j}\right\| ;
\end{aligned}
$$

hence the mapping $\Sigma_{j} f_{j} g_{j} h_{j} \rightarrow \Sigma_{j} f_{j} \otimes g_{j} \otimes h_{j}$ is an isometry which may be extended to an isometric isomorphism from $B$ onto $R Z(S) \otimes S A P(S) \otimes L Z(S)$.

3.11. Remarks. (a) If $f \in L M C(S)$ and $Z=\mathrm{Cl}(R(S) f) \cup\{f\}$ (pointwise closure), then it is easily shown that $f \in M D(S)$ if and only if the flow $(S, Z, \pi)$ is distal, where $\pi(s)=\left.R(s)\right|_{z}$.

(b) The following simple examples illustrate some of the extreme behavior that can occur in the theory of distal functions on semigroups. If $S$ is a right zero semigroup then $S D(S)=D(S)=C(S)$, and $M D(S)=\mathrm{C}$, the space of constant functions. For a left zero semigroup $M D(S)=D(S)=C(S)$, and $S D(S)=\mathrm{C}$. If $S$ is a null semigroup then $D(S)=C(S)$ and $S D(S)=M D(S)=\mathrm{C}$. If $S$ is the direct product of a left zero semigroup $L$ and a right zero semigroup $R$ then $r s t=r t$ for all $r, s, t \in S$; hence the $L M C$ compactification of $S$ has property (b) of Lemma 3.3 and therefore $D(S)=L M C(S)$. Also, the $S D$ and $M D$ compactifications of $S$ are right zero and left zero semigroups respectively; hence $S D(S)=R Z(S)=\mathrm{C} \otimes C(R)$ and $M D(S)=L Z(S)=C(L) \otimes C$. Note that in this case $D(S)$ may be properly contained in $C(S)$. (Take $L=[0,1]$ with the usual topology and $R$ the set of nonnegative integers with the discrete topology. If $f(t, n)=t^{n}$ then $f \notin L M C(S)$.)

(c) Theorem 3.4 may be used to obtain the universal properties of various other distal compactifications of a semigroup $S$. For example, the $M D \cap S D L U C$ compactification $(X, \alpha)$ of $S$ is maximal with respect to the property that $X$ is a group and the mapping $(s, x) \rightarrow \alpha(s) x: S \times X \rightarrow X$ is continuous, and the $D \cap W A P$ compactification $(Y, \beta)$ of $S$ is maximal with respect to the property that $Y$ is a semitopological semigroup and an inflation of a rectangular group. Note that in the latter compactification, the mappings $f_{e}, g_{e}$ and $h_{e}$ of Lemma 3.3 are continuous. 
However, the inflation function $r: Y \rightarrow K(Y)$ need not be continuous as the following example shows: Let $S$ be the compact semitopological semigroup constructed in [2, p. 156]. Since $u v w=u w$ for all $u, v, w$ in $S, S$ must be its own $D \cap W A P$ compactification. On the other hand, $K(S)$ is not closed; hence $r$ cannot be continuous.

(d) It is interesting to compare the algebra of distal functions on a semitopological semigroup $S$ with the algebra of what we shall call proximal functions. A function $f \in L M C$ is proximal if for each pair $g, h \in \mathrm{Cl}(R(S) f)$ there exists a net $\left(s_{n}\right)$ in $S$ such that $\lim _{n} R\left(s_{n}\right) g=\lim _{n} R\left(s_{n}\right) h$. Using methods similar to those used in the proof of Lemma 3.2 one may show that $f$ is proximal if and only if $x y(f)=x z(f)$ for all $x \in K(X)$ and all $y, z \in X$, where $X$ denotes the $L M C(S)$ compactification of $S$. It follows that the set $\Pi(S)$ of proximal functions on $S$ is an admissible subalgebra of $C(S)$ and that the $\Pi$ compactification $(Y, \beta)$ of $S$ is maximal with respect to the property that $K(Y)$ is a left zero semigroup. Theorem 3.4 then implies that $\Pi(S) \cap M D(S)=L Z(S)$.

4. Extensions of distal functions. Throughout this section $S$ denotes a dense subsemigroup of a semitopological semigroup $T$. We shall consider the following extension problem: Let $F$ denote any of the prefixes $D, S D, M D, D L U C, S D L U C$ or $M D L U C$. When does each member of $F(S)$ extend to a member of $F(T)$ ? Equivalently: If $(X, \alpha)$ is an $F$ compactification of $T$, when is $\left(X,\left.\alpha\right|_{S}\right)$ an $F$ compactification of $S$ ?

In general, a distal function on $S$ need not even extend to a continuous function on $T$. As an example, let $T=[0, \infty)$ with the usual topology and ordinary multiplication and let $S$ be the subgroup $(0, \infty)$. Since $T$ has an identity its $D$ compactification $(X, \alpha)$ is a group. But $\alpha(0)$ is a zero of $X$; hence $X=\{\alpha(0)\}$ and therefore $D(T)$ consists only of constant functions. Since $D(S)$ has a rich supply of nonconstant functions, $\left.D(S) \nsubseteq C(T)\right|_{s}$. Note that in this example $t S \cap S=\varnothing$ for $t=0$. In Theorem 4.5 we show that, in general, if $t S \cap S \neq \varnothing$ for all $t \in T$ then $M D(S) \cap$ $S D(S)=\left.M D(T) \cap S D(T)\right|_{S}$.

4.1. LemMA. Let $S_{1}$ and $S_{2}$ be semitopological semigroups and $\theta: S_{1} \rightarrow S_{2}$ a continuous homomorphism. Let $F$ denote any of the prefixes $D, S D, M D, D L U C$, $S D L U C$, or MDLUC. Then $\theta^{*}\left(F\left(S_{2}\right)\right) \subset F\left(S_{1}\right)$. In particular, taking $\theta: S \rightarrow T$ to be the inclusion mapping, we have $\left.F(T)\right|_{S} \subset F(S)$.

Proof. It is easily verified that $\theta^{*}\left(\operatorname{LMC}\left(S_{2}\right)\right) \subset L M C\left(S_{1}\right)$ and $\theta^{*}\left(\operatorname{LUC}\left(S_{2}\right)\right) \subset$ $\operatorname{LUC}\left(S_{1}\right)$. Let $\left(X_{i}, \alpha_{i}\right)$ denote the canonical $L M C$ compactification of $S_{i}(i=1,2)$. Consider $\theta^{*}$ as a map from $\operatorname{LMC}\left(S_{2}\right)$ to $L M C\left(S_{1}\right)$ and let $\psi$ denote the restriction of $\theta^{* *}$ to $X_{1}$. Then $\psi \circ \alpha_{1}=\alpha_{2} \circ \theta$ and hence $\psi: X_{1} \rightarrow X_{2}$ is a homomorphism. If $g \in D\left(S_{2}\right)$ and $u, v, e^{2}=e \in X_{1}$ then by 3.2(i) applied to $g$ we have (uev) $\left(\theta^{*}(g)\right)=$ $\psi(u e v)(g)=\psi(u v)(g)=(u v)\left(\theta^{*}(g)\right)$. Therefore $\theta^{*}(g) \in D\left(S_{1}\right)$. The other cases are proved in a similar manner.

4.2. Lemma. Let $F$ denote any of the prefixes $D, S D$, or $M D$. If $g \in L M C(T)$ and $f=\left.g\right|_{S} \in F(S)$ then $g \in F(T)$. 
Proof. Let $(X, \alpha)$ and $(Y, \beta)$ denote the $L M C$ compactifications of $S$ and $T$ respectively, and let $\theta: S \rightarrow T$ denote the inclusion mapping. Consider $\theta^{*}$ as a mapping from $\operatorname{LMC}(T)$ to $\operatorname{LMC}(S)$. Then $\theta^{*}(g)=f$, and $\psi=\left.\theta^{* *}\right|_{X}$ is a continuous homomorphism from $X$ onto $Y$. Let $u, v, e^{2}=e \in Y$ and choose $x, y \in X$ and $d \in E(X)$ such that $\psi(x)=u, \psi(y)=v$ and $\psi(d)=e$. Then if $f \in D(S),(u e v)(g)$ $=\psi(x d y)(g)=(x d y)(f)=(x y)(f)=(u v)(f)$, so $g \in D(T)$ by 3.2(i). The other cases are proved in a similar manner.

4.3. TheOREM. Let $T$ be a semitopological group and suppose that $S$ has the finite intersection property (f.i.p.) for right ideals. Then $\operatorname{DLUC}(S)=S D L U C(S)=$ $M D L U C(S)=\left.D L U C(T)\right|_{s}$.

Proof. Let $f \in D L U C(S)$. By Corollary 3.3 of [10] there exists $g \in L U C(T)$ such that $\left.g\right|_{S}=f$. By 4.2 then $g \in D L U C(T)$. Therefore $\left.D L U C(S) \subset D L U C(T)\right|_{s}$. Also, by 3.5 and 4.1, DLUC(T) $\left.\right|_{S}=\left.M D L U C(T)\right|_{S} \subset M D L U C(S)$, and similarly for $S D L U C(S)$. This completes the proof.

4.4. Lemma. Suppose $t S \cap S \neq \varnothing$ for all $t \in T$. If $g \in C(T)$ and $f=\left.g\right|_{S} \in S D(S)$ (respectively, $f \in S D L U C(S)$ ) then $g \in S D(T)$ (respectively, $g \in S D L U C(T)$ ).

Proof. By 4.2 it suffices to show that $g \in L M C(T)$ (respectively, $g \in L U C(T)$ ). For this it is enough to show that if $\left(s_{n}\right)$ is a net in $S$ converging to $t \in T$ then $w\left(L\left(s_{n}\right) g\right) \rightarrow w(L(t) g)$ for each (respectively, uniformly in) $w \in \operatorname{spec}(C(T))$ (see, for example, Lemma 2.2 of [13]). Let $(Y, \beta)$ denote the $S D$ (respectively, $S D L U C$ ) compactification of $S$, and let $\left(\beta\left(s_{m}\right)\right)$ be a subnet of $\left(\beta\left(s_{n}\right)\right)$ which converges to some $y \in Y$. If $r \in S$ is chosen so that $t r \in S$ we have

$$
\beta(t r)=\lim _{m} \beta\left(s_{m} r\right)=y \beta(r),
$$

and for any $s \in S$,

$$
L(t) g(s)=\lim _{m} g\left(s_{m} s\right)=\lim _{m} \beta\left(s_{m} s\right)(f)=[y \beta(s)](f) .
$$

Now, by 3.4, $Y$ is right simple; hence we may choose $e \in E(Y)$ and $z \in Y$ such that $\beta(r) z=e$. Given $w \in \operatorname{spec}(C(T))$, let $\left(r_{k}\right)$ be a net in $S$ and $u \in Y$ such that $w(h)=\lim _{k} h\left(r_{k}\right)(h \in C(T))$ and $z \beta\left(r_{k}\right) \rightarrow u$. By (1) and (2),

$$
\begin{aligned}
w(L(t) g) & =\lim _{k} g\left(t r_{k}\right)=\lim _{k}\left[y \beta\left(r_{k}\right)\right](f) \\
& =\lim _{k}\left[y \beta(r) z \beta\left(r_{k}\right)\right](f) \\
& =\lim _{k}\left[\beta(t r) z \beta\left(r_{k}\right)\right](f) \\
& =[\beta(t r) u](f) .
\end{aligned}
$$

Similarly, for each $n, w\left(L\left(s_{n}\right) g\right)=\left[\beta\left(s_{n} r\right) u\right](f)$. Since $\left[\beta\left(s_{n} r\right) u\right](f)$ converges to $[\beta(t r) u](f)$ for each $u \in Y$ (respectively, uniformly in $u \in Y$ ), it follows that $w\left(L\left(s_{n}\right) g\right) \rightarrow w(L(t) g)$ for each (respectively, uniformly in) $w \in \operatorname{spec}(C(T))$, as required. 
4.5. Theorem. (a) If $t S \cap S \neq \varnothing$ and $S t \cap S \neq \varnothing$ for all $t \in T$, then $S D(S)=$ $\left.S D(T)\right|_{s}$ and $S D L U C(S)=\left.S D L U C(T)\right|_{S}$.

(b) If $t S \cap S \neq \varnothing$ for all $t \in T$ then $S D(S) \cap M D(S)=\left.S D(T) \cap M D(T)\right|_{S}$ and $\operatorname{SDLUC}(S) \cap \operatorname{MDLUC}(S)=\left.\operatorname{SDLUC}(T) \cap M D L U C(T)\right|_{s}$.

Proof. We prove only (a); the proof of the other part is similar. By 4.1 and 4.4 it is enough to show that if $f \in S D(S)$ then there exists $g \in C(T)$ such that $\left.g\right|_{s}=f$. For this it is enough to show that if $\left(r_{m}\right)$ and $\left(s_{n}\right)$ are nets in $S$ converging to $t \in T$ such that the limits $a=\lim _{m} f\left(r_{m}\right)$ and $b=\lim _{n} f\left(s_{n}\right)$ exist then $a=b$. Let $(Y, \beta)$ denote the canonical $S D$ compactification of $S$. We may suppose that the limits $x=\lim _{m} \beta\left(r_{m}\right)$ and $y=\lim _{n} \beta\left(s_{n}\right)$ exist in $Y$. Choose $r \in S$ such that $r t \in S$. Then $\beta(r) x=\lim _{m} \beta\left(r r_{m}\right)=\beta(r t)=\lim _{n} \beta\left(r s_{n}\right)=\beta(r) y$. Since $Y$ is right simple it is left cancellative; hence $x=y$. In particular, $a=x(f)=y(f)=b$, as required.

We shall say that a flow $(S, Z, \pi)$ extends to the flow $(T, Z, \bar{\pi})$ if $\left.\bar{\pi}\right|_{S}=\pi$. The following theorem gives the connection between extending distal flows and extending distal functions.

4.6. TheOREM. Let $S$ be a dense subsemigroup of a semitopological semigroup $T$. Then $S D(S) \cap M D(S)=\left.S D(T) \cap M D(T)\right|_{S}$ (respectively, $S D L U C(S) \cap$ $\left.M D L U C(S)=\left.S D L U C(T) \cap M D L U C(T)\right|_{S}\right)$ if and only if every point transitive distal (respectively, point transitive jointly continuous distal) flow $(S, Z, \pi)$ extends to a flow $(T, Z, \bar{\pi})$ of the same type.

Proof. We shall prove the $L U C$ version. Let $F(S)=S D L U C(S) \cap M D L U C(S)$ and define $F(T)$ analogously. Suppose $F(S)=\left.F(T)\right|_{s}$. Then $\theta^{*}$, the dual of the inclusion map $\theta: S \rightarrow T$, is an isometric isomorphism from $F(T)$ onto $F(S)$. Let $(X, \alpha)$ and $(Y, \beta)$ denote, respectively, the $F$ compactifications of $S$ and $T$ and set $\gamma=\left.\theta^{* *}\right|_{X}$, where $\theta^{*}$ is considered as a map on $F(T)$ to $F(S)$. Then $\gamma: X \rightarrow Y$ is a topological isomorphism such that $\gamma \circ \alpha=\beta \circ \theta$. Let $(S, Z, \pi)$ be any point transitive jointly continuous distal flow with $z_{0} \in Z$ such that $\mathrm{Cl}\left(S z_{0}\right)=Z$. Then $(E(S, Z), \pi)$ is a right topological compactification of $S, E(S, Z)$ is a group, and the mapping $(s, \xi) \rightarrow \pi(s) \circ \xi$ from $S \times E(S, Z)$ to $E(S, Z)$ is continuous. Therefore, by Remark 3.11(c), there exists a continuous homomorphism $\psi: X \rightarrow E(S, Z)$ such that $\psi \circ \alpha=\pi$. Let $\bar{\pi}$ denote the continuous homomorphism $\psi \circ \gamma^{-1} \circ \beta$ : $T \rightarrow E(S, Z)$. Then $\bar{\pi} \circ \theta=\psi \circ \gamma^{-1} \circ \beta \circ \theta=\psi \circ \alpha=\pi$. Hence, if we show that $(t, z) \rightarrow \bar{\pi}(t)(z)$ is continuous on $T \times Z$ to $Z$, then $(T, Z, \bar{\pi})$ will be a flow of the required type (distal because $E(T, Z)=E(S, Z)$ ). Now, since $E(S, Z)\left(z_{0}\right)=Z$ and $\psi \circ \gamma^{-1}: Y \rightarrow E(S, Z)$ is a surjection, there exists for each $z \in Z$ a member $y \in Y$ such that

$$
z=\left[\psi \circ \gamma^{-1}(y)\right]\left(z_{0}\right)
$$

Then if $t \in T$,

$$
\begin{aligned}
\bar{\pi}(t)(z) & =\left[\left(\psi \circ \gamma^{-1}\right)(\beta(t))\right](z) \\
& =\left[\left(\psi \circ \gamma^{-1}\right)(\beta(t))\right] \circ\left[\left(\psi \circ \gamma^{-1}\right)(y)\right]\left(z_{0}\right) \\
& =\left[\left(\psi \circ \gamma^{-1}\right)(\beta(t) y)\right]\left(z_{0}\right) .
\end{aligned}
$$


Since the mapping $(t, y) \rightarrow \beta(t) y: T \times Y \rightarrow Y$ is continuous, it follows from (2) that $\{\bar{\pi}(\cdot)(z): z \in Z\}$ is an equicontinuous family of mappings on $T$ to $Z$. Thus $(t, z) \rightarrow \bar{\pi}(t)(z)$ will be continuous if we show that for any $t \in T$ the mapping $\bar{\pi}(t)$ : $Z \rightarrow Z$ is continuous. To this end let $\left(z_{n}\right)$ be a net in $Z$ converging to $z$ and let $\left(\bar{\pi}(t)\left(z_{k}\right)\right)$ be a convergent subnet of $\left(\bar{\pi}(t)\left(z_{n}\right)\right)$. For each $k$ choose, according to (1), $y_{k} \in Y$ such that $z_{k}=\left[\psi \circ \gamma^{-1}\left(y_{k}\right)\right]\left(z_{0}\right)$. We may assume that $y_{k} \rightarrow y \in Y$. Then $z=\left[\psi \circ \gamma^{-1}(y)\right]\left(z_{0}\right)$, and from (2) we see that $\bar{\pi}(t)\left(z_{k}\right) \rightarrow \bar{\pi}(t)(z)$. Therefore $\bar{\pi}(t)(z)$ is the unique limit point of $\left(\bar{\pi}(t)\left(z_{n}\right)\right)$; hence $\bar{\pi}(t)$ is continuous.

Conversely, suppose that the stated extension property for flows holds, and let $(X, \alpha)$ be as above. Define $\pi: S \rightarrow X^{X}$ by $\pi(s)(x)=\alpha(s) x(s \in S, x \in X)$. Then $(S, X, \pi)$ is a jointly continuous flow with dense orbit $\pi(S)(e)$, where $e$ is the identity of the group $X$. Also, $(S, X, \pi)$ is clearly distal. Therefore $(S, X, \pi)$ extends to a flow $(T, X, \bar{\pi})$. Given $f \in F(S)$ define $g \in C(T)$ by $g(t)=[\bar{\pi}(t)(e)](f)$. Then $\left.g\right|_{S}=f$, and by 4.2 the proof will be complete once we show that $g \in L U C(T)$. Now, for any $s \in S$ and $t \in T$,

$$
L(t) g(s)=[\bar{\pi}(t)(\alpha(s))](f)
$$

as is easily checked. Also, since the mapping $(t, x) \rightarrow \bar{\pi}(t)(x): T \times X \rightarrow X$ is continuous and $X$ is compact, $\{\bar{\pi}(\cdot)(x): x \in X\}$ is an equicontinuous family. It follows from (3) that $L(\cdot) g$ is norm continuous on $T$.

5. Distal compactifications of semidirect products. Let $S$ and $T$ be semigroups with identities (each denoted by 1 ) and let $\tau: T \times S \rightarrow S$ be a mapping with the following properties: $\tau(t, \cdot)$ is a homomorphism such that $\tau(t, 1)=1, \tau(1, \cdot)$ is the identity mapping, and $\tau(r t, \cdot)=\tau(r, \tau(t, \cdot))(r, t \in T)$. If multiplication on the set $S \times T$ is defined by

$$
(s, t)\left(s^{\prime}, t^{\prime}\right)=\left(s \tau\left(t, s^{\prime}\right), t t^{\prime}\right)
$$

then $S \times T$ becomes a semigroup with identity $(1,1)$ called a semidirect product of $S$ and $T$ and denoted by $S(\tau) T$. If $S$ and $T$ are semitopological semigroups we shall assume that $S(T) T$ is also a semitopological semigroup (i.e. that $\tau$ is spearately continuous and $(s, t) \rightarrow s \tau\left(t, s^{\prime}\right)$ is continuous for each $\left.s^{\prime} \in S\right)$.

5.1. TheOREM. Let $Q=S(T) T$ be a semidirect product of the semitopological semigroups $S$ and $T$, and suppose that $S$ is compact. Let $(Y, \beta)$ be the DLUC compactification of $T$. Then there exist a right topological compactification $(X, \alpha)$ of $S$ and a semidirect product $Z=X$ (P) $Y$ such that $(Z, \alpha \times \beta)$ is a DLUC compactification of $Q$. Furthermore, $X$ is a continuous homomorphic image of the SAP compactification of $S$ and hence is a topological group.

Proof. Let $p_{1}: Q \rightarrow S$ and $p_{2}: Q \rightarrow T$ denote the projection mappings and define $q_{1}: S \rightarrow Q$ and $q_{2}: T \rightarrow Q$ by $q_{1}(s)=(s, 1), q_{2}(t)=(1, t)$. Since $S$ is compact it is easy to see that $f(S, \cdot)$ is norm compact in $C(T)$ for each $f \in L U C(Q)$. Furthermore, since $q_{1}, q_{2}$, and $p_{2}$ are continuous homomorphisms, we have from Lemma 4.1 that $q_{2}^{*}(D L U C(Q))=D L U C(T), \quad\left(q_{2} \circ p_{2}\right)^{*}(D L U C(Q)) \subset D L U C(Q), \quad$ and 
$q_{1}^{*}(D L U C(Q)) \subset D L U C(S)=S A P(S)$, the last equality by 3.6. Therefore by Theorem 3.1 of [11] the proof will be complete once we show that, for $f \in D L U C(Q)$, the function $g=\left(q_{1} \circ p_{1}\right)^{*}(f)$ is also in $D L U C(Q)$.

We show first that $g \in L U C(Q)$. Let $\left(s_{n}, t_{n}\right)$ be a net in $Q$ converging to $\left(s_{0}, t_{0}\right)$, and let $(Z, \gamma)$ denote the canonical $D L U C$ compactification of $Q$. Let $z_{n}=\gamma\left(1, t_{n}\right)^{-1}$ and $z_{0}=\gamma\left(1, t_{0}\right)^{-1}$ (inverses in the group $Z$ ). If $u$ is any limit point of $\left(z_{n}\right)$, then by the joint continuity of the mapping $(q, z) \rightarrow \gamma(q) z: Q \times Z \rightarrow Z, \gamma\left(1, t_{0}\right) u$ is a limit point of $\gamma\left(1, t_{n}\right) z_{n}=\gamma(1,1)$. Therefore $z_{n} \rightarrow z_{0}$. Now, for any $(s, t) \in Q$,

$$
\begin{aligned}
\left|L\left(s_{n}, t_{n}\right) g(s, t)-L\left(s_{0}, t_{0}\right) g(s, t)\right|=\left|f\left(s_{n} \tau\left(t_{n}, s\right), 1\right)-f\left(s_{0} \tau\left(t_{0}, s\right), 1\right)\right| \\
=\left|\hat{f}\left(\gamma\left(s_{n}, t_{n}\right) \gamma(s, 1) z_{n}\right)-\hat{f}\left(\gamma\left(s_{0}, t_{0}\right) \gamma(s, 1) z_{0}\right)\right| \\
\leqslant\left|\hat{f}\left(\gamma\left(s_{n}, t_{n}\right) \gamma(s, 1) z_{n}\right)-\hat{f}\left(\gamma\left(s_{0}, t_{0}\right) \gamma(s, 1) z_{n}\right)\right| \\
+\left|\hat{f}\left(\gamma\left(s_{0}, t_{0}\right) \gamma(s, 1) z_{n}\right)-\hat{f}\left(\gamma\left(s_{0}, t_{0}\right) \gamma(s, 1) z_{0}\right)\right|,
\end{aligned}
$$

where $\hat{f} \in C(Z)$ and $\gamma^{*}(\hat{f})=f$. The first term on the right of this inequality is dominated by $\left\|L\left(s_{n}, t_{n}\right) f-L\left(s_{0}, t_{0}\right) f\right\|$ and hence tends to zero uniformly in $s \in S$. The second term also tends to zero uniformly in $s$ because $S$ is compact and $z_{n} \rightarrow z_{0}$. Thus $g \in \operatorname{LUC}(Q)$.

Let $(W, \delta)$ denote the canonical $L U C$ compactification of $Q$. To show that $g$ is distal it suffices by Lemma 3.2 to show that if $e \in E(W)$ and $w \in W$ then $T_{e w} g=T_{w} g$. Let $\left(s_{n}, t_{n}\right)$ and $\left(a_{m}, b_{m}\right)$ be nets in $Q$ such that $\delta\left(s_{n}, t_{n}\right) \rightarrow w$ and $\delta\left(a_{m}, b_{m}\right) \rightarrow e$. We may assume that $s_{n} \rightarrow s_{0}$ and $a_{m} \rightarrow a_{0}$ in $S$, and that $\delta\left(1, b_{m}\right) \rightarrow u$ in $W$. We shall construct a sequence $\left\{c_{k}\right\}$ in $S$ such that for all positive integers $k$

$$
\delta\left(c_{k}, 1\right) u^{k}=e
$$

For $k=1$ we may take $c_{k}=a_{0}$. If $c_{k}$ has been defined, let $c_{k+1}$ be any limit point of $\left(a_{0} \tau\left(b_{m}, c_{k}\right)\right)$ in $S$. Then $e=e^{2}=e \delta\left(c_{k}, 1\right) u^{k}=\lim _{m} \delta\left(a_{0}, b_{m}\right) \delta\left(c_{k}, 1\right) u^{k}=$ $\lim _{m} \delta\left(a_{0} \tau\left(b_{m}, c_{k}\right), 1\right) \delta\left(1, b_{m}\right) u^{k}=\delta\left(c_{k+1}, 1\right) u u^{k}$. Since the closure of $\left\{u^{k}: k=\right.$ $1,2, \ldots\}$ is a compact subsemigroup of $W$ it contains an idempotent $d$; hence by (1), $e=\delta(c, 1) d$ for some $c \in S$. Note that $d \in \mathrm{Cl}(\delta(1, T))$ so there exists a net $\left(r_{j}\right)$ in $T$ such that $\delta\left(1, r_{j}\right) \rightarrow d$. Now for any $(s, t) \in Q$,

$$
\begin{aligned}
\left(T_{w} g\right)(s, t) & =\lim _{n} g\left((s, t)\left(s_{n}, t_{n}\right)\right) \\
& =\lim _{n} f\left(s \tau\left(t, s_{n}\right), 1\right) \\
& =\lim _{n} \hat{f}\left(\gamma(s, t) \gamma\left(s_{n}, 1\right) \gamma(1, t)^{-1}\right) \\
& =\hat{f}\left(\gamma(s, t) \gamma\left(s_{0}, 1\right) \gamma(1, t)^{-1}\right)
\end{aligned}
$$


and

$$
\begin{aligned}
\left(T_{e w} g\right)(s, t)= & {[\delta(s, t) \delta(c, 1) d w](g) } \\
= & \lim _{j} \lim _{n}\left[\delta(s, t) \delta(c, 1) \delta\left(1, r_{j}\right) \delta\left(s_{n}, t_{n}\right)\right](g) \\
= & \lim _{j} \lim _{n} g\left(s \tau(t, c) \tau\left(t r_{j}, s_{n}\right), t r_{j} t_{n}\right) \\
= & \lim _{j} \lim _{n} f\left(s \tau(t, c) \tau\left(t r_{j}, s_{n}\right), 1\right) \\
= & \lim _{j} \lim _{n} \hat{f}\left(\gamma(s, t) \gamma(c, 1) \gamma\left(1, r_{j}\right)\right. \\
& \left.\times \gamma\left(s_{n}, 1\right) \gamma\left(1, r_{j}\right)^{-1} \gamma(1, t)^{-1}\right) \\
= & \lim _{j} \hat{f}\left(\gamma(s, t) \gamma(c, 1) z_{j} \gamma(1, t)^{-1}\right),
\end{aligned}
$$

where $z_{j}=\gamma\left(1, r_{j}\right) \gamma\left(s_{0}, 1\right) \gamma\left(1, r_{j}\right)^{-1}$. Let $\theta: W \rightarrow Z$ denote the restriction mapping. Since $\theta$ is a homomorphism and $Z$ is a group, $\gamma(1,1)=\theta(e)=\theta(\delta(c, 1) d)=$ $\gamma(c, 1) \theta(d)=\gamma(c, 1)$; hence

$$
\left(T_{e w} g\right)(s, t)=\lim _{j} \hat{f}\left(\gamma(s, t) z_{j} \gamma(1, t)^{-1}\right)
$$

Comparing this expression with the one for $\left(T_{w} g\right)(s, t)$ we see that all that is needed yet is to show that $\gamma\left(s_{0}, 1\right)$ is a limit point of the net $\left(z_{j}\right)$. Let $\left(\tau\left(r_{i}, s_{0}\right)\right)$ be a subnet of $\left(\tau\left(r_{j}, s_{0}\right)\right)$ converging to $p \in S$. Then since $z_{j}=\gamma\left(\tau\left(r_{j}, s_{0}\right), 1\right), \gamma(p, 1)$ is a limit point of $\left(z_{j}\right)$. On the other hand, since $\gamma\left(1, r_{j}\right)=\theta\left(\delta\left(1, r_{j}\right)\right) \rightarrow \theta(d)=\gamma(1,1)$, we have $\gamma(p, 1)=\lim _{i} \gamma\left(\tau\left(r_{i}, s_{0}\right), 1\right) \gamma\left(1, r_{i}\right)=\lim _{i} z_{i} \gamma\left(1, r_{i}\right)=\lim _{i} \gamma\left(1, r_{i}\right) \gamma\left(s_{0}, 1\right)=$ $\gamma\left(s_{0}, 1\right)$. This completes the proof.

5.2. RemARKS. (a) If $S$ is not compact then the DLUC compactification of $S(\tau) T$ need not be a semidirect product, even if $T$ is a compact topological group. As an example, let $Q$ be the Euclidean group $\mathbf{C}(\tau) T$, where $\mathbf{C}$ is the group of complex numbers, $T$ is the circle group and $\tau(w, z)=w z(w \in T, z \in \mathbf{C})$. Let $f(z, w)=$ $\exp \left(i \operatorname{Re}\left(z w^{-1}\right)\right)$. We shall show that $f \in D L U C(Q)$ but $\left(q_{1}^{\circ} p_{1}\right)^{*} \notin D L U C(Q)$. By Theorem 3.1 of [11] this will show that the DLUC compactification of $Q$ cannot be a semidirect product. Let $\left(z_{n}, w_{n}\right) \rightarrow\left(z_{0}, w_{0}\right)$ in $Q$. Then for all $(z, w) \in Q$

$$
\begin{aligned}
& \left|L\left(z_{n}, w_{n}\right) f(z, w)-L\left(z_{0}, w_{0}\right) f(z, w)\right| \\
& \quad=\left|\exp \left(i \operatorname{Re}\left(z_{n}\left(w_{n} w\right)^{-1}+z w^{-1}\right)\right)-\exp \left(i \operatorname{Re}\left(z_{0}\left(w_{0} w\right)^{-1}+z w^{-1}\right)\right)\right| \\
& \quad \leqslant 2\left|\operatorname{Re}\left(z_{n}\left(w_{n} w\right)^{-1}+z w^{-1}\right)-\operatorname{Re}\left(z_{0}\left(w_{0} w\right)^{-1}+z w^{-1}\right)\right| \\
& \quad \leqslant 2\left|z_{n} w_{n}^{-1}-z_{0} w_{0}^{-1}\right|
\end{aligned}
$$

hence $f \in L U C(Q)$. To see that $f$ is distal, note first that every member of the pointwise closure of $R(Q) f$ is of the form $R(0, w)(u f)$ for some $u, w \in T$. If $\left(z_{k}, w_{k}\right)$ is a net in $Q$ such that $\lim _{k} R\left(z_{k}, w_{k}\right) R(0, w)(u f)=\lim _{k} R\left(z_{k}, w_{k}\right) R\left(0, w^{\prime}\right)\left(u^{\prime} f\right)$ then $R\left(0, w w^{\prime \prime}\right)\left(u u^{\prime \prime} f\right)=R\left(0, w^{\prime} w^{\prime \prime}\right)\left(u^{\prime} u^{\prime \prime} f\right)$ for some $u^{\prime \prime}, w^{\prime \prime} \in T$; hence 
$R(0, w)(u f)=R\left(0, w^{\prime}\right)\left(u^{\prime} f\right)$. Therefore $f \in D L U C(Q)$. On the other hand, if $\left(w_{n}\right)$ is a sequence in $T$ with $\operatorname{Re}\left(w_{n}\right)=\pi(2 n)^{-1}$, and if $z$ is real, then $L\left(0, w_{n}\right) g(z, w)=$ $\cos \left(z \pi(2 n)^{-1}\right)+i \sin \left(z\left(\pi(2 n)^{-1}\right)\right)$, which obviously cannot converge uniformly in $z$. (Note. P. Milnes has given general conditions on a semidirect product $S(\tau) T$ of two topological groups which ensure that a function $f$ of the form $f(s, t)=$ $h\left(\tau\left(t^{-1}, s\right)\right)$ is distal whenever $h \in D L U C(S)$ [15]. Our construction is a special case of his result.)

(b) If $S$ and $T$ are semitopological semigroups with right and left identities respectively, then the $D \cap A P$ compactification of the direct product $S \times T$ is the direct product of the $D \cap A P$ compactifications of $S$ and $T$. This follows from Theorem 1 of [9]. The analogous results hold for the $S D \cap A P$ and $M D \cap A P$ cases as well. However, unless at least one of $S$ or $T$ is compact, the $D L U C$ compactification of $S \times T$ need not be a direct product, as the following example shows: Let $S=T=I$, the group of integers, and let $\theta: I \times I \rightarrow I$ denote the homomorphism $(m, n) \rightarrow m+n$. The function $g: I \rightarrow \mathbf{C}$ defined by $g(n)=\exp \left(i n^{2}\right)$ is distal but not almost periodic [12]. By 4.1, $f=\theta^{*}(g)$ is distal on $I \times I$. If the distal compactification of $I \times I$ were a direct product then the set of functions $f(I, \cdot)$ would be relatively norm compact (see, for example, Theorem 1 of [9]). But then $g$ would be almost periodic since $f(I, \cdot)=R(I) g$.

(c) The wreath product construction generates an interesting class of semidirect products to which Theorem 5.1 may be applied. Let $U$ be a compact topological semigroup with identity and $T$ a discrete semigroup with identity such that $T$ acts on the right on a set $J$, and let $S=U^{J}$ with coordinatewise multiplication and the product topology. Define $\tau: T \times S \rightarrow S$ by $\tau(t, s)(j)=s(j t)$, where $j t$ denotes the action of $t$ on $j$. The semidirect product $Q=S$ (T) $T$ is called a wreath product of $U$ and $T$. According to Theorem 5.1 the $D L U C$ compactification of $Q$ is a semidirect product $X \subseteq Y$, where $(Y, \beta)$ is a $D L U C$ compactification of $T$ and $(X, \alpha)$ is a continuous homomorphic image of the $S A P$ compactification of $U^{J}$ (which may be written as $Z^{J}$, where $Z$ is the $S A P$ compactification of $U$ (see [9])). (For $A P$ and $S A P$ compactifications of wreath products, see [11].)

(d) Under the hypotheses of 5.1 we have $\operatorname{DLUC}(S \odot T)=A \otimes D L U C(T)$, where $A$ is an admissible subalgebra of $D L U C(S)$. This follows from the identifications $D L U C(S \odot T)=C(X \odot Y)=C(X) \otimes C(Y)=\alpha^{*}(C(X)) \otimes \beta^{*}(C(Y))$.

\section{REFERENCES}

1. L. Auslander and F. Hahn, Real functions coming from flows on compact spaces and concepts of almost periodicity, Trans. Amer. Math. Soc. 106 (1963), 415-426.

2. J. F. Berglund and K. H. Hofmann, Compact semitopological semigroups and weakly almost periodic functions, Lecture Notes in Math., vol. 42, Springer-Verlag, Berlin and New York, 1967.

3. J. F. Berglund, H. D. Junghenn and P. Milnes, Compact right topological semigroups and generalizations of almost periodicity, Lecture Notes in Math., vol. 663, Springer-Verlag, Berlin and New York, 1978.

4. K. deLeeuw and I. Glicksberg, Applications of almost periodic compactifications, Acta. Math. 105 (1961), 63-97.

5. R. Ellis, Lectures on topological dynamics, Benjamin, New York, 1969.

6. L_ Locally compact transformation groups, Duke Math. J. 24 (1957), 119-126.

7. H. Furstenberg, The structure of distal flows, Amer. J. Math. 85 (1963), 477-515. 
8 K. H. Hofmann and P. S. Mostert, Elements of compact semigroups, Merrill, Columbus, Ohio, 1966.

9. H. D. Junghenn, $C^{*}$-algebras of functions on direct products of semigroups, Rocky Mountain J. Math. 10 (1980), 589-597.

10. __ Extensions of continuous functions on dense semigroups, Illinois J. Math. (to appear)

11. H. D. Junghenn and B. T. Lerner, Semigroup compactifications of semidirect products, Trans. Amer. Math. Soc. 265 (1981), 393-404.

12. A. W. Knapp, Distal functions on groups, Trans. Amer. Math. Soc. 128 (1967), 1-40.

13. A. T. Lau, Invariant means on dense subsemigroups of topological groups, Canad. J. Math. 23 (1971), 797-801.

14. P. Milnes, Compactifications of semitopological semigroups, J. Austral. Math. Soc. 15 (1973), 488-503.

15. , Minimal and distal functions on semidirect products of groups, preprint.

16. I. Namioka, Right topological groups, distal flows, and a fixed point theorem, Math. Systems Theory 6 (1972), 193-209.

17. W. Ruppert, Rechtstopologische Halbgruppen, J. Reine Angew. Math. 261 (1973), 123-133.

18. M. Petrich, Introduction to semigroups, Merrill, Columbus, Ohio, 1973.

Department of Mathematics, George Washington University, Washington, D. C. 20052 